\title{
Douleurs neuropathiques : quelles avancées cliniques et thérapeutiques
}

\section{Neuropathic pain : clinical and therapeutic advances}

\author{
N. Attal · D. Bouhassira \\ C) Springer-Verlag France 2010
}

Ce dossier spécial de Douleur et analgésie aborde les aspects cliniques les plus actuels des douleurs neuropathiques douleurs associées à une lésion ou à un dysfonctionnement du système nerveux. Ces douleurs sont fréquentes, puisque leur prévalence en population générale en France a récemment été estimée à $7 \%$, et à $5 \%$ pour les douleurs neuropathiques modérées à sévères [1]. Leur impact sur la qualité de vie et leur coût pour la société sont considérables [2]. Si la recherche fondamentale est déterminante notamment pour mieux en élucider les mécanismes et développer des traitements actifs sur de nouvelles cibles, nous avons souhaité ici mettre l'accent sur plusieurs avancées cliniques dans le domaine des douleurs neuropathiques qui nous paraissent essentielles. Ces avancées incluent l'« explosion » des outils diagnostiques et d'évaluation de ces douleurs dont les applications à la pratique clinique ou à la recherche ne cessent de s'étendre ; la place accrue dans leur évaluation - y compris en dehors de la recherche — des outils de mesure sophistiqués comme l'évaluation quantitative des troubles sensitifs et les techniques électrophysiologiques ; l'attention croissante accordée aux facteurs psychologiques, qui semblent aussi prédictifs de la chronicité douloureuse ; l'élaboration tant au niveau national qu'international de recommandations thérapeutiques fondées sur les preuves dans le traitement pharmacologique de ces douleurs ; enfin le développement de nouvelles stratégies thérapeutiques non médicamenteuses allant de la stimulation magnétique transcrânienne à la stimulation électrique du système nerveux, dont les applications ne cessent de s'étendre.

Le dynamisme de la recherche clinique en la matière est aussi illustré par le fait qu'un grand nombre des chapitres de ce dossier ont été rédigés par de jeunes chercheurs, français ou étrangers. Que les auteurs de ces chapitres, jeunes ou moins jeunes, soient ici vivement remerciés pour leur participation et leur motivation dans ce domaine.

\section{Références}

1. Bouhassira D, Lantéri-Minet M, Attal N, et al (2008) Prevalence of chronic pain with neuropathic characteristics in the general population. Pain 136:380-7

2. Doth AH, Hansson PT, Jensen MP, Taylor RS (2010) The burden of neuropathic pain: a systematic review and meta-analysis of health utilities. Pain [Epub ahead of print] 